\title{
Simulation of Three-fold Symmetric Photonic Crystal Structures on Top of GaN LEDs
}

\author{
Simeon Trieu*, Xiaomin Jin", Chang Xiong ${ }^{* *}$, Xingxing Fu ${ }^{* *}$, Xiangning Kang ${ }^{* *}$, Guoyi Zhang ${ }^{* *}$ and \\ Bei Zhang \\ *Electrical Engineering Department, 1 Grand Avenue, California Polytechnic State University, San Luis Obispo, CA 93407 \\ **State Key Laboratory for Mesoscopic Physics, Physics Department, Peking University, Beijing, China 100871 \\ Author e-mail address: xjin@calpoly.edu
}

\begin{abstract}
A 3-fold symmetric photonic-crystal grating is simulated using improved FDTD-model. Transmission gratings are optimized. Then, the best cases are simulated in GaN-LED models. The maximum extraction efficiency improvement is 40x greater compared to conventional LEDs. (C)2010 Optical Society of America

OCIS codes: (140.3325) Laser coupling; (050.5298) Photonic crystals
\end{abstract}

\section{Introduction}

Light emitting diodes (LED) have found many applications such as residential and commercial lighting, fiber optics, machine vision, and colored displays. These applications demand low power, high efficiency, high luminosity, and low heat generation. To fulfill these necessary conditions, a grating structure is the solution by creating more angles of escape and also diffraction mechanisms. Grating structures can be patterned with many shapes including: pyramidal, spherical, conical, cylindrical, and so on, but only a few can be fabricated with great success. For example, with the modified laser lift off (M-LLO) technique, holes can be patterned at a 4 micron period instead of etching a random structure. In the experiment at Peking University (PKU), grating depths varied from $75 \mathrm{~nm}$ to $120 \mathrm{~nm}[1]$.

Using a 3-D finite difference time domain (FDTD) method, a 3PC grating structure is first optimized and characterized to observe trends for optimizations for certain grating cell-to-cell radii, widths, and heights. Then the optimized grating parameters from a transmission grating are entered into an entire GaN device model. The current literature does not provide optimizations for full 3-D GaN models, only grating simulations. Comparisons between the best case grating parameters are analyzed to verify optimal parameters and discover modeling trends.

\section{Simulation Model}

The simulations use a 3-D FDTD method [2-3] for a GaN LED model to calculate final output power. In all simulations, a time monitor is placed in 5 locations: top, front, back, left side, and right side. This allows for a complete picture of the total power output radiating from all facets. After the simulation time has elapsed, the final average output power is taken from each monitor and summed into a total output power measured in arbitrary units (au). Note that a bottom monitor is unnecessary since either the reflections are unimportant, as in the case of a grating-only simulation, or the Ag reflector plate is expected to reflect the vast majority waves back towards the one of the other monitors, as in the full LED model. For thin metal reflectors, only a negligible amount of radiation is transmitted as evanescent waves.

There are two models in this simulation set. The first simulation, a simple semiconductor-air model, aids in discovering the optimized grating parameters. A smaller model is necessary to sweep the parameters since a 3-D FDTD simulation's simulation time and memory requirements expand with simulation domain size as $\mathrm{N}^{4}$ and $\mathrm{N}^{3}$, respectively. Therefore, the device size is limited to the amount of RAM and reasonable time we have per simulation. The first simulation has a duration of 500fs to observe the transmission properties of each grating configuration. A transmission grating carved out of the semiconductor surface that can be varied by three variables: grating cell-to-cell radius $(A)$, grating cell width $(w)$, and grating height $(d)$. We step through the possible combinations that are physically allowed (ie. $\mathrm{w} \leq \mathrm{A}$ ). For example, the first case would be $\mathrm{A}=1 \mu \mathrm{m}, \mathrm{w}=1 \mu \mathrm{m}, \mathrm{d}=800 \mathrm{~nm}$, then $\mathrm{A}=1 \mu \mathrm{m}, \mathrm{w}=1 \mu \mathrm{m}, \mathrm{d}=1000 \mathrm{~nm}$, and so on. Light waves are generated from random spatial and directional current sources to better represent incoherent light. 32 current sources are placed at random intervals throughout the source layer to provide a pseudorandom distribution. The simulation results in a good representation of a photonic crystal's response to light from all incident angles (ie. 0 to 90 degrees). This is a new way to setup light sources, which gives grating huge efficiency improvement results compared to other simulation models [2-3], and is a more comprehensive model for the $3 \mathrm{PC}$ simulation. 


\section{JTuD33.pdf}

For the second simulation, a full GaN LED model aids in understanding a diffraction grating's response to a multi-layered thin film LED with a Ag reflector plate. The optimal grating parameters from the first simulation are used in this simulation as the grating parameters for the transmission grating. The grating is carved from the undoped GaN layer. Only the top 5 cases from the grating optimization simulations are taken into account for comparison. In this second simulation, the simulation time is increased to $2500 \mathrm{fs}$ since we need to account for multiple reflections from the $\mathrm{Ag}$ reflector plate in this case.

\section{Results}

The grating optimization simulations revealed that a transmission grating is optimized when $\mathrm{A}=\mathrm{w}, \mathrm{n}$ other words, when unit cells acquire the most area on the LED surface. In recent literature, this is known as a filling factor and can be described as the ratio of the total area covered by the unit cells and the total top surface area. Fig. 1 shows the results of the 3-D FDTD, limited to the interface between GaN to air. A grating with the parameters $A, w$, and $d$, was varied in steps. It is clear that as the grating height increases, the total output power also increases. The improvement, in all cases of $\mathrm{A}=1,3$, and $6 \mu \mathrm{m}$, at $\mathrm{d}=800 \mathrm{~nm}$ indicates that the relationship between $d$ and output power may have a sinusoidal-like relationship.

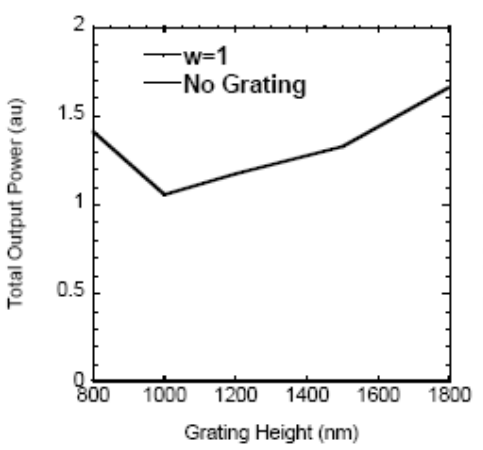

(a)



(b)

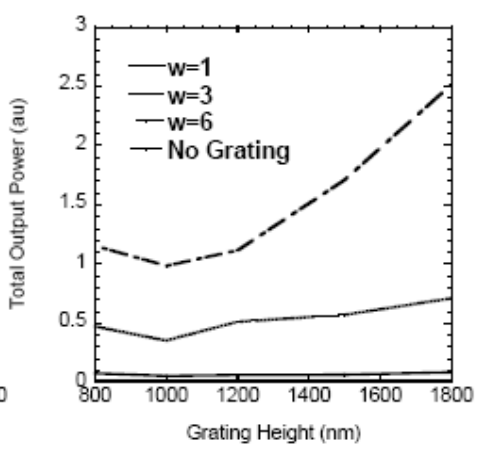

(c)

Fig.1 Total output power for a GaN-to-Air grating structure: (a) $\mathrm{A}=1 \mu \mathrm{m}$., (b) $\mathrm{A}=3 \mu \mathrm{m}$., and (c) $\mathrm{A}=6 \mu \mathrm{m}$.

Next, a 3-D FDTD full GaN LED model was simulated with the best cases of the grating-only simulations. These best cases and their results for the full GaN model are shown in Table 1. The grating with the characteristics of $\mathrm{A}=3 \mu \mathrm{m}, \mathrm{w}=3 \mu \mathrm{m}, \mathrm{d}=1800 \mathrm{~nm}$ produces the best transmission grating. Even though the $A=6 \mu \mathrm{m}, \mathrm{w}=6 \mu \mathrm{m}, \mathrm{d}=1800 \mathrm{~nm}$ grating had the best results in the grating-only simulations, the results in the full GaN LED model indicate that it performed the worst out of all 5 gratings simulated. Grating-only simulations are not sufficient indicators of optimized grating structures when considering full GaN LED models. Internal modes must be considered as well as multiple reflections from any reflectors.

Table 1 - Full GaN Model 3-D FDTD Simulations for Best Case Gratings

\begin{tabular}{|c|c|c|c|c|c|}
\hline A (um) & w (um) & d (nm) & $\begin{array}{l}\mathbf{P}\left(|\mathbf{E}|^{2}, \mathbf{a u ) ~ o f ~ t h e ~}\right. \\
\text { Grating-Only }\end{array}$ & $\begin{array}{l}\mathbf{P}\left(|\mathbf{E}|^{2}, \mathbf{a u}\right) \text { of the Full } \\
\text { GaN Model }\end{array}$ & $\begin{array}{l}\text { Improvement factor for Full } \\
\text { GaN Model }\end{array}$ \\
\hline 3 & 3 & 1800 & 2.010 & 43.292 & 43.77 \\
1 & 1 & 1800 & 1.658 & 42.381 & 42.83 \\
3 & 3 & 1500 & 1.651 & 41.165 & 41.57 \\
6 & 6 & 1500 & 1.703 & 40.807 & 41.20 \\
6 & 6 & 1800 & 2.508 & 40.359 & 40.74 \\
\hline \multicolumn{7}{|r|}{} \\
\hline
\end{tabular}

\section{References}

[1] Bao, K., Kang, X., Zhang, B., Dai, T., Sun, Y., Fu, Q., Lian, G., Xiong, G., Zhang, G. and Chen, Y., "Improvement of light extraction from GaN-based thin-film light-emitting diodes by patterning undoped GaN using modified laser lift-off," Appl. Phys. Lett. 92, 141104 (2008).

[2] Yee, K., "Numerical solution of initial boundary value problems involving Maxwell's equations in isotropic media", IEEE Trans. Antennas and Propagation 14, 302-307 (1966).

[3] Taflove, A., Brodwin, M. E. "Numerical solution of steady-state electromagnetic scattering problems using the time-dependent Maxwell's equations", IEEE Trans. Microwave Theory and Techniques 23. 623-630 (1975).

\section{Acknowledgments}

This project is supported by the NSF 08-603 OISE - EAPSI 2009 fellowship award and ONR 7-N000140811209. 